\title{
A new III-V Nanowire-Quantum Dot Single Photon Source with Improved Purcell Factor for Quantum Communication
}

shahramm mohammad nejad ( $\sim$ shahramm@iust.ac.ir)

Iran University of Science and Technology https://orcid.org/0000-0002-1818-3023

Amine Mahmoudi

Iran University of Science and Technology School of Electrical Engineering

Hossein Arab

Iran University of Science and Technology School of Electrical Engineering

\section{Research Article}

Keywords: quantum dot, nanowire, single photon source, Purcell factor, Quality factor, telecommunication

Posted Date: June 1st, 2021

DOI: https://doi.org/10.21203/rs.3.rs-530578/v1

License: (c) (i) This work is licensed under a Creative Commons Attribution 4.0 International License.

Read Full License 


\title{
A new III - V Nanowire-Quantum Dot Single Photon Source with improved Purcell factor for quantum communication
}

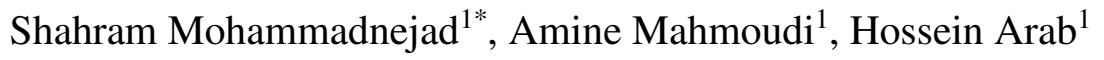 \\ ${ }^{1}$ Nanoptronics Research Centre (NRC), School of Electrical Engineering, Iran University of Science \\ \& Technology (IUST), Tehran, Iran \\ *Corresponding Author Email: shahramm@iust.ac.ir
}

\begin{abstract}
:
In this work, the finite difference time domain (FDTD) method has been utilized to simulate the propagation emission from $\mathrm{PbS}$ quantum dots in a hexagonal InP nanowire as a single photon source. The effect of height and radius of the nanowire as well as the location and orientation of the dipole source in the Purcell factor and Quality factor of the nanowire have been investigated. A broadband electric dipole source has been used to model the quantum dot and the effect of shape and radius of $\mathrm{PbS}$ quantum dot have been investigated in the final results. The conclusive structure has been optimized to a nanowire with hexagonal cross section with radius of $220 \mathrm{~nm}$ and height of 10um. The emission peak obtained above 1um with Purcell factor of 4.72 which is in good agreement with cases have been used as single photon source in quantum communication.

Keywords: quantum dot; nanowire; single photon source; Purcell factor; Quality factor; telecommunication.
\end{abstract}




\section{Introduction}

Recently, high-speed and reliable data transferring and processing have attracted researchers' attention in the field of quantum information technology. In recent studies, one of the typical candidates for single and entangled photon sources, are semiconductor quantum dots (QDs) [1]. In this way, self-assembled semiconductor QDs created by Stranski-Kranstanov (SK) [2-4] method, have good potential to be applied in practical systems of light sources. However, their solid state environment brings up some challenges [5].

In order to produce entangled photon pairs, QDs with high structural symmetry are needed that is one of the fundamental technologies in quantum processing. While self-assembled QDs have been utilized widely, but their symmetry is significantly low, not only in the direction perpendicular to the substrate, although in the horizontal direction due to the adatom diffusion length variations in substrate direction. Hence, many studies have been done in order to find some methods to improve structural symmetry. Temperature control, the use of magnetic fields [6] and growth with strain-reducing layers [7, 8] are some of the mentioned methods in the literature [1].

Recently, nanowires (NWs) have received researchers' attention due to their special optical and electronic characteristics. They have been of great interest in nano-sized electronic and optoelectronic devices because of strong two dimensional confinement that they cause in electrons, holes and photons [9]. Additionally, for efficient production of single photons, quantum emitter has been coupled to a resonance mode of a cavity or NW waveguide with high quality factor [10]. Single photon emission from QDs coupled to NWs has been widely studied for wavelengths lower than 1um [11-14]. Furthermore, during recent years some cases of NW-QD structures with the emission wavelengths above $1 \mathrm{um}$ has been reported to be applied in telecommunications $[15,16]$. 
These types of structures are suitable candidates for the light sources because of their doping, shape and material controllability [15].

In another side, QDs can be produced in a flask by chemical methods. These types of QDs which have been called colloidal QDs (CQDs), have high three-dimensional symmetry [1]. Also, because CQDs like PbS and PbSe cover a wide range of wavelengths [17], these QDs can be used in optical telecommunication wavelengths. Moreover, their production cost also is low [1]. For these reasons, CQDs are attractive for the optoelectronics and also the quantum information technology.

QD position control is crucial to achieve an ideal QD-NW coupled system. The position of the SK QDs is controlled using a processed substrate, but this method seems undesirable, because the preprocessing destroys the quantum efficiency by producing crystal defects. While to control the position of CQDs, a nano-hole is made using screening probe microscopy (SPM) on the $\mathrm{Si}$ substrate to trap a PbS CQD. SPM position control technology has the maximum accuracy of single atom, and of course this method can be used to integrate devices with Si photonic technology [1].

Despite recent developments, another serious limitation of IR sources based on QDs for utilizing in telecommunications, is their low modulation speed. Regardless of the electrical constraints, the main limitation of the modulation speed is the low spontaneous emission rate of the $\mathrm{PbS}$ QDs, which have a typical lifetime about a few microseconds. This low radiative recombination rate has been attributed to the unconventional highly degenerated band structure of QDs or to the large dielectric screening of excitons in lead-based salts. The mechanism, however, is not entirely clear. 
To solve the problem of low lifetime of Lead-based QDs, they have been integrated with waveguides, especially the optical cavities with low mode volume to improve the radiative characteristics of emitters by Purcell effect. Purcell effect can be defined as increasing the radiative and non-radiative transition rates of quantum emitters by increasing the local density of optical states in the location of emitter [18]. Furthermore, one of the most common approaches to improve extraction efficiency of single photon sources is utilizing Purcell effect to resonate excitonic emission with guiding mode of NW. Two factors that are needed for this aim are high Quality factor and low Volume mode which have been expressed as a factor of Q/V [19].

First demonstration of coupling CQDs to photonic crystal cavities has been showed by Fushman et al. in 2005. They used PMMA-soluble PbS QDs deposited on cavities with AlGaAs membrane as a wide-band on-chip source to characterize cavities [17]. In another investigation that has been done by Akselrod et al. in 2016 in the field of IR light emitting diodes, the emission lifetime of QDs has been decreased from microsecond to nanosecond by coupling PbS QDs to colloidal plasmonic nanoantennas based on metal nanocubes, which has been done by increasing radiative decay rate and finally, it led to an increase in the quantum efficiency of the nanoantenna [18]. Additionally, there have been done a lot of studies in recent years on PbS/CdS core/shell QDs [20, 21].

Many studies have been performed on the effect of the in-plane point dipole position whose polarization is perpendicular to the axis of the NW. This situation is observed experimentally in high-efficiency single photon sources (SPSs) based on a self-assembled QD in a photonic wire. Assuming that the lateral confinement is not important (as in the behavior of an axial quantum well), a QD can be modeled with an in-plane dipole source in a wurtzite material [22]. 
Although FDTD is a classic calculator, it can be a tool for optimizing optical devices and components of quantum photon experiments. One of the major design challenges in this field is the optimization of light collection from solid state QDs, which are called SPSs and are usually achieved through nanopillar cavities.

It is not possible to model a single photon in a classical way, however an ideal SPS produces completely uncorrelated photons, so it can be modeled using an incoherent non-polar source and how many photons on average Collected by fiber can be measured.

In this paper, for the first time it has been attempted to simulate a PbS CQD inside a hexagonal InP NW as a single photon source to use in quantum communication, to obtain the optimum Purcell factor, quality factor, and electric field intensity of the emitted mode with respect to the emitted wavelength per radius and height of the NW, shape and size of the QD as well as the location of QD along the NW axis. In this work, the NW material had to be selected in such a way that the band gap of the NW was larger than the band gap of the QD material to achieve the quantum confinement of the QD. To do this, InP NWs have been utilized whose lattice constant is close to that of the $\mathrm{PbS}$ as presented in Figure 1.

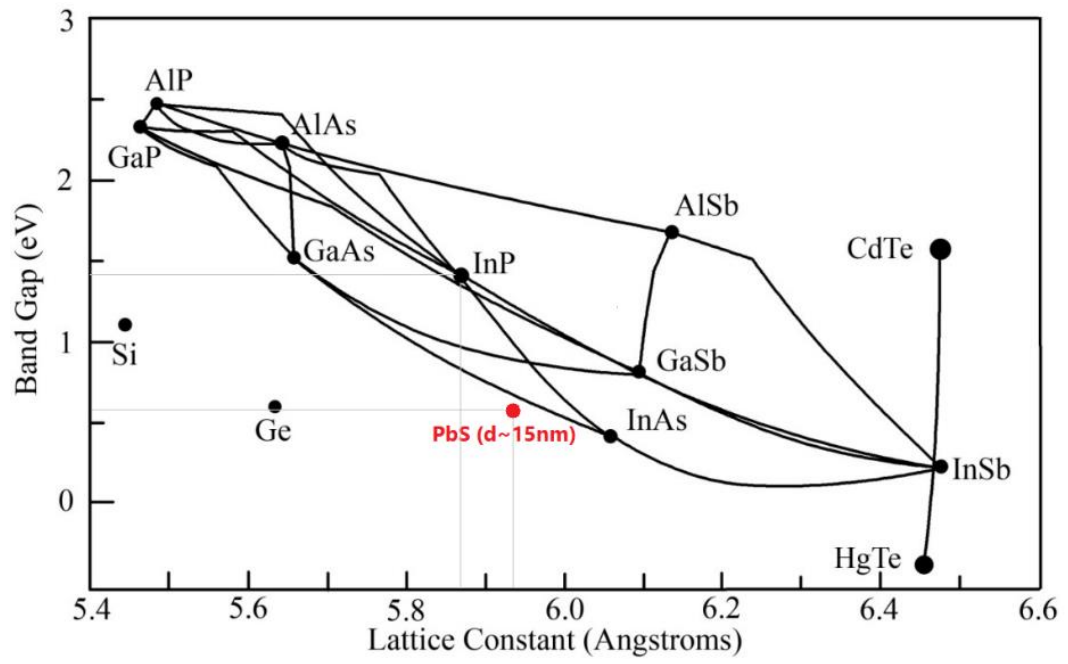

Figure 1: band gap diagram versus lattice constant for some semiconductor materials [23]. Properties for PbS QDs with diameter of $15 \mathrm{~nm}$ have been added (red circle) [24]. 


\section{NW modeling}

A NW is inherently a Fabry-Perot cavity with two surfaces that act as reflectors. Semiconductor NWs Due to their crystalline structure, have a non-circular cross section, which makes it very difficult to solve Maxwell equations for waveguide modes inside the NW. For this reason, by approximating the hexagonal cross section instead of the circle, it is possible to obtain low-order waveguide modes with good accuracy. In 2005, Nobis and Grundmann [25] by considering similar refractive indices for both circular and hexagonal NW structures, expressed the scaling relationship of their cross-sectional surfaces as follows:

$$
\frac{3 \sqrt{3}}{2} L^{2}=\pi a^{2}
$$

In this relation, $a$ is the radius of the circular NW and $L$ is the face size of the hexagonal NW, which is: $1.1 a=L$.

Within the non-absorption spectrum, the waveguide properties of NWs can be retrieved by solving Maxwell equations as follows:

$$
\begin{aligned}
& \left(\nabla^{2}+n^{2} k^{2}-\beta^{2}\right) \vec{e}=0 \\
& \left(\nabla^{2}+n^{2} k^{2}-\beta^{2}\right) \vec{h}=0
\end{aligned}
$$

Where $k=2 \pi / \lambda ; \lambda$ is the wavelength of light in vacuum and $\beta$ is the propagation constant. These equations can be solved with the following eigenvalue equations in cylindrical coordinates for the modes $H E_{v m}$ and $E H_{v m}$ as follows:

$$
\left\{\frac{J_{v}^{\prime}(U)}{U J_{v}(U)}+\frac{K_{v}^{\prime}(W)}{W K_{v}(W)}\right\}\left\{\frac{J_{v}^{\prime}(U)}{U J_{v}(U)}+\frac{n_{2}^{2} K_{v}^{\prime}(W)}{n_{1}^{2} W K_{v}(W)}\right\}=\left(\frac{v \beta}{k n_{1}}\right)^{2}\left(\frac{V}{U W}\right)^{4}
$$

In this equation, $J_{v}$ and $K_{v}$ are Bessel functions. $U=a\left(k_{0}^{2} n_{1}^{2}-\beta^{2}\right)^{1 / 2}, W=a\left(\beta^{2}-k_{0}^{2} n_{2}^{2}\right)^{1 / 2}$ and $V=a k_{0}\left(n_{1}^{2}-n_{2}^{2}\right)^{1 / 2}$ are waveguide parameters [26]. 
By solving these equations numerically, NW waveguide modes can be obtained. When the diameter of the NW is less than the wavelength of the guided light, new properties are created in the NW that lead to severe light limitation inside it and the light can be easily conducted in it. It also exhibits a single-mode performance for a specified value of NW diameter, and usually occurs when the NW diameter is less than $\lambda / n_{1}$ [24]. However, if the NW is too thin, it will show poor reflection and will have a lot of leakage losses.

\section{Simulation results}

In this paper, NWs with hexagonal cross-section and substrates of the same material as InP have been used. Although circular and hexagonal NWs are almost identical, hexagonal NWs eliminate degeneracy of $H E_{11}$ mode due to reduced structural symmetry. Also, relatively low reflection from the end face, limits the Q-factor (which is the stored energy divided by the energy dissipation per cycle) of F-P cavity semiconductor NW to below a few hundred.

In addition, although semiconductor NWs have very low surface roughness, they usually have very high waveguide losses (e.g. $\mathrm{SnO}_{2}$ nanoribbon, $1-8 \mathrm{~dB} / \mathrm{mm}$ in the $500 \mathrm{~nm}$ wavelength range [27]) compared to glass nanofibers (e.g. $\mathrm{SiO}_{2}$ nanofiber, $0.1 \mathrm{~dB} / \mathrm{mm}$ in the $600 \mathrm{~nm}$ wavelength range [28]). In this work, the wavelength of the dipole source has been set to be broadband from 830 to $1420 \mathrm{~nm}$. The losses for the $H E_{11}$ mode for the finally structured NW are $0.13 \mathrm{~dB} / \mathrm{cm}$. More losses for other guiding modes have been obtained compared to $H E_{11}$ mode. Given that, for nanophotonic applications, the effective length required for semiconductor NWs is usually a few ten micrometers, losses at this level are acceptable.

Due to the difference in the arrangement of the atoms in the two types of crystal structures of zinc-blend and wurtzite, zinc-blend NWs emit light with polarization along the NW axis, while in wurtzite NWs, the emitted light has polarization perpendicular to the growth axis of the NW. In 
the simulations, we have modeled the single-photon emission from the QD with an electric dipole located on the NW axis, which allows dipole orientation as shown in Figure 2. In fact, the radiative properties of a quantum emitter can be considered using the classical electromagnetic effect, assuming that the quantum emitter operates as a point dipole with time-varying dipole moment. The dipole source in FDTD can be used to study these radiative properties in a homogeneous or a non-uniform environment where emission is affected by scattering and absorption of light by near dipole structures.

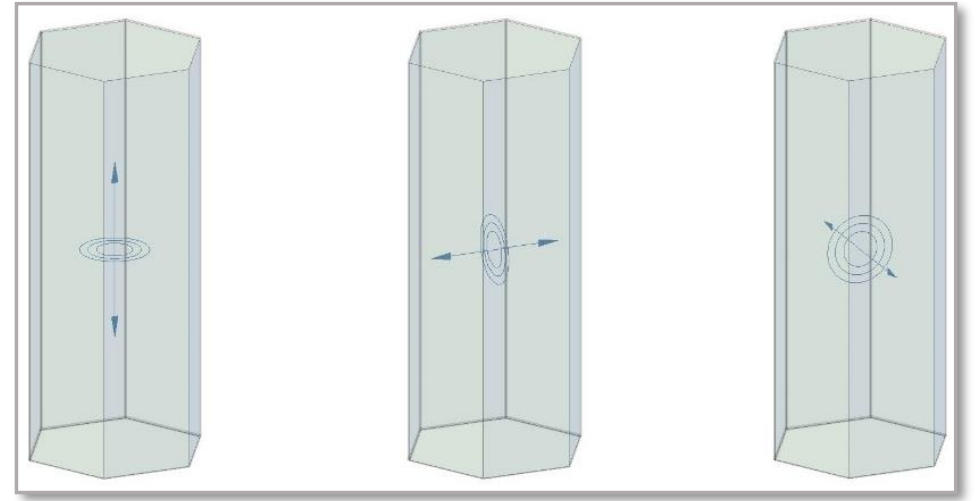

a)

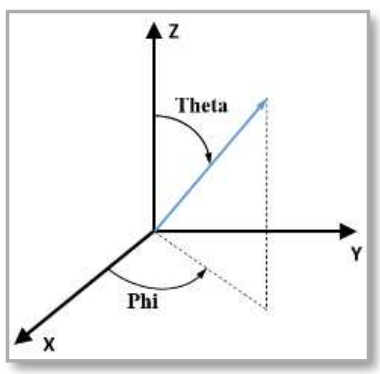

b)

Figure 2: a) Illustrations of various types of dipole source orientation within the NW structure. b) Theta and phi angles.

The angular distribution of the power emitted by a dipole placed in vacuum, is a function of $\sin ^{2} \phi$, which for the $\phi=0$, orientation, is in the dipole's orientation. Therefore, it is expected that light is emitted perpendicular to the NW axis and no light is observed in the direction parallel to the dipole, however, due to the dielectric environment around the dipole, the emission profile is modified.

The intensity of the electric field is also a function of the light transverse k-vector, which is perpendicular to the direction of photon emission (Figure 3). Also, the emission angle $\phi$ as $k=$ $\arcsin \phi$ is related to the $k$-vector. The minimum and maximum electric field intensities per $\mathrm{k}=0$ 
are obtained when the dipole is oriented parallel to the NW axis and perpendicular to the NW axis. Purcell factor and quality factor are examined by changing the dipole source polarization angles in FDTD. According to the diagram in Figure 4(a), the maximum value for both factors is obtained when the polarization of the dipole source is perpendicular to the NW axis and located along the $\mathrm{x}$-axis. In addition, the highest electric field intensity is obtained for the same condition shown in Figure 4(b).

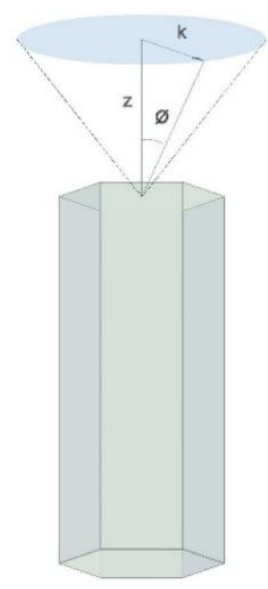

Figure 3: Light transverse k-vector and $\phi$ angle.

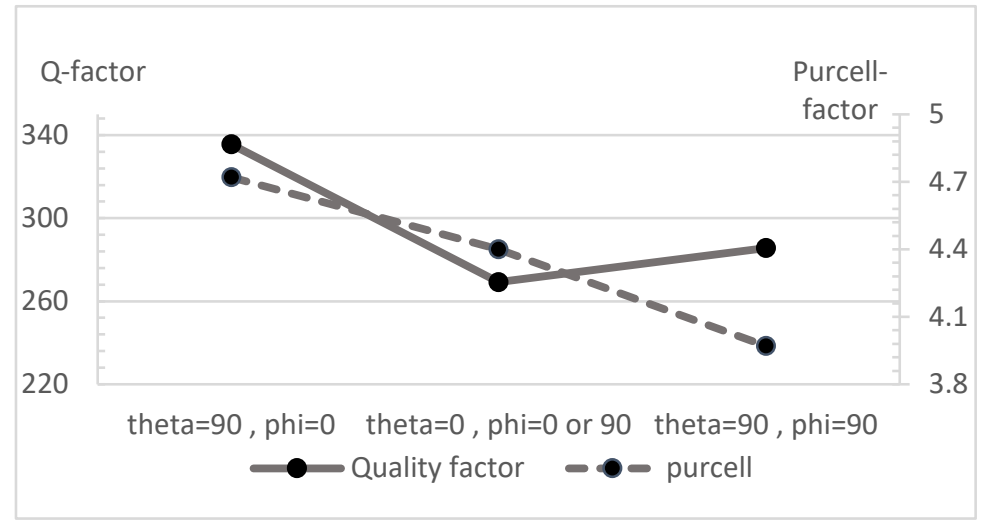

a)

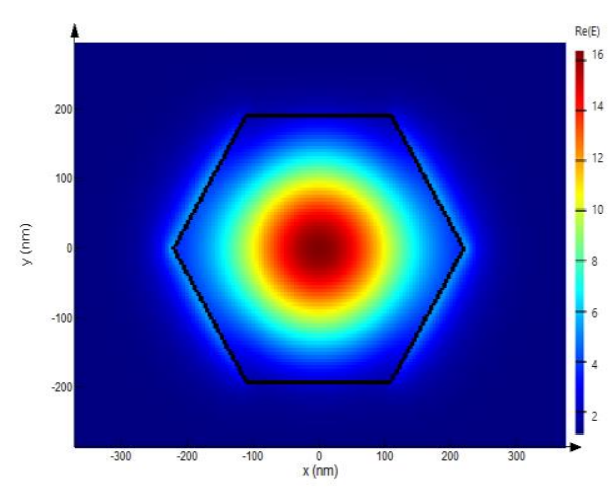

b)

Figure 4: a) Quality factor and Purcell factor versus variation of polarization angle of dipole source. b) The intensity of electric field per theta $=90$ and $p h i=0$. 
Figure 5 shows the quality factor and the Purcell factor based on the changes in the radius of the NW for a dipole source located at the center of the NW with a height of 10 um and orientation perpendicular to its axis. Peak wavelengths are always above $1000 \mathrm{~nm}$ for all radii. As it can be seen in the Figure 5, at low radii, for a radius of $160 \mathrm{~nm}$, a peak is observed in Purcell factor and quality factor. The peak wavelength obtained for a radius of $160 \mathrm{~nm}$ is equal to $1047 \mathrm{~nm}$; However, the intensity of the electric field observed at this peak is weak. As the NW radius increases from about $180 \mathrm{~nm}$, both factors increase.

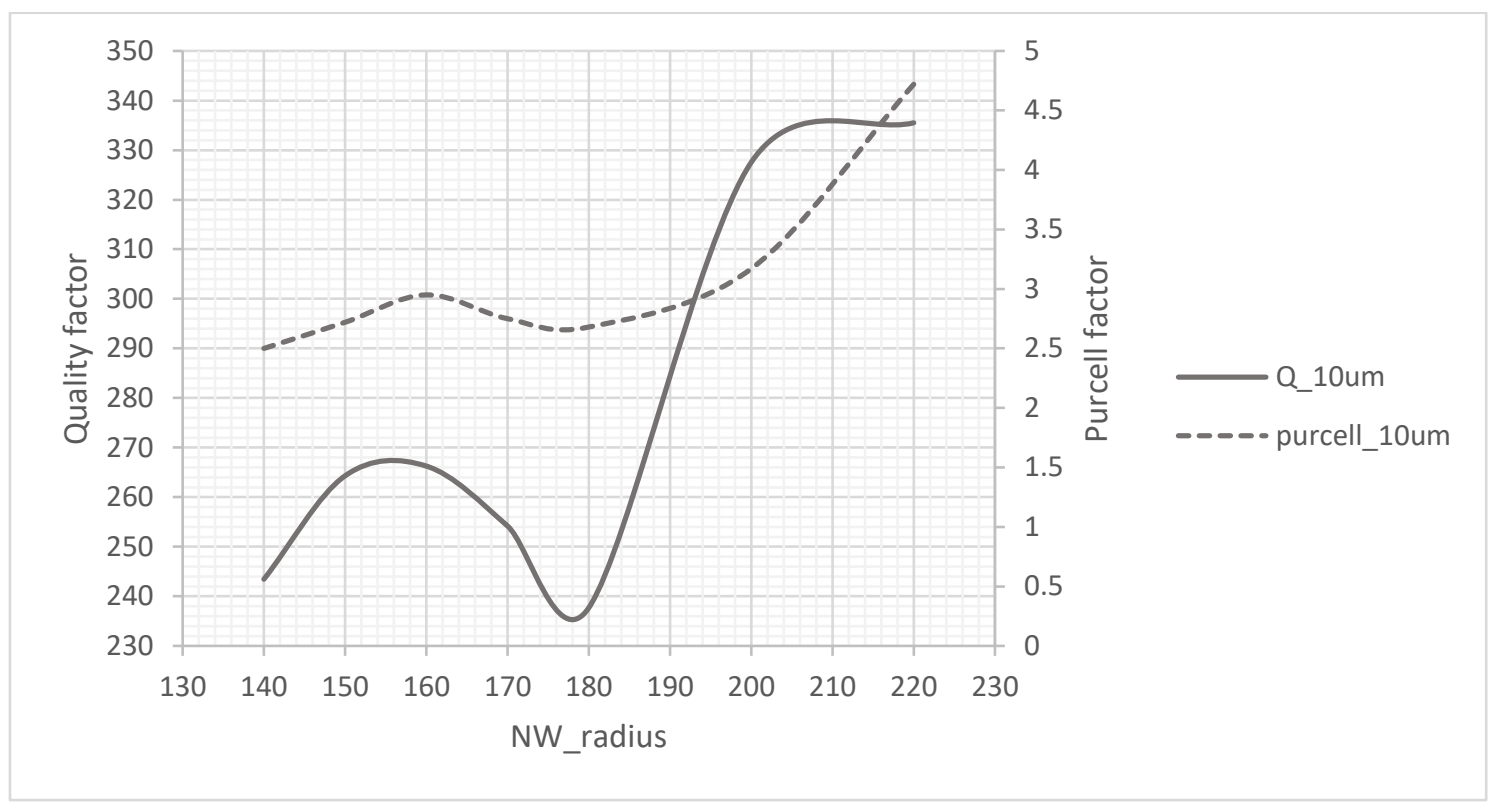

Figure 5: Purcell factor and Quality factor versus variation of NW radius (the NW height is considered to be 10um). 


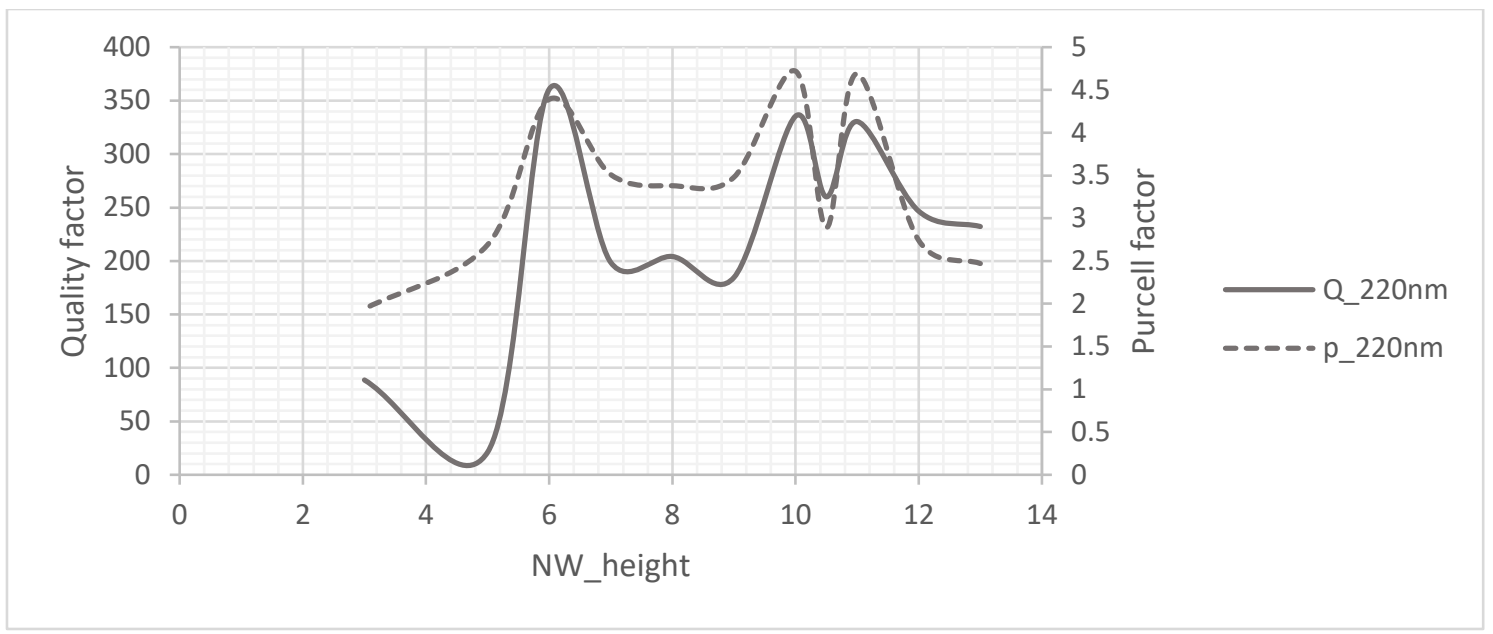

Figure 6: Purcell factor and Quality factor versus variation of the height of the NW (the NW radius is considered to be $220 \mathrm{~nm}$ ).

In the following, the effect of sweeping the NW height from 3 um to 13 um on quality factor and Purcell factor is examined (Figure 6). Maximum Purcell factor which observed, is strongly dependent on the NW height. In this structure, as the height of the NW increases, the peaks of the structure shift to higher wavelengths. So that for NW heights lower than 8 um, the wavelength of the maximum Purcell factor is less than $1000 \mathrm{~nm}$. With increasing NW height from 8 um onwards, two peaks can be seen in Figure 6, obtained for 10um and 11 um heights, respectively, and their Purcell factor and quality factor values difference is insignificant.

According to the diagrams in Figure 5 and Figure 6, the values of radius and height of NW was selected as $220 \mathrm{~nm}$ and $10 \mathrm{um}$, respectively. Afterwards, the profile of electric field intensity is obtained as shown previous in Figure 4(b). The selected guiding mode in the NW was $H E_{11}$ mode which has been shown in Figure 7. 


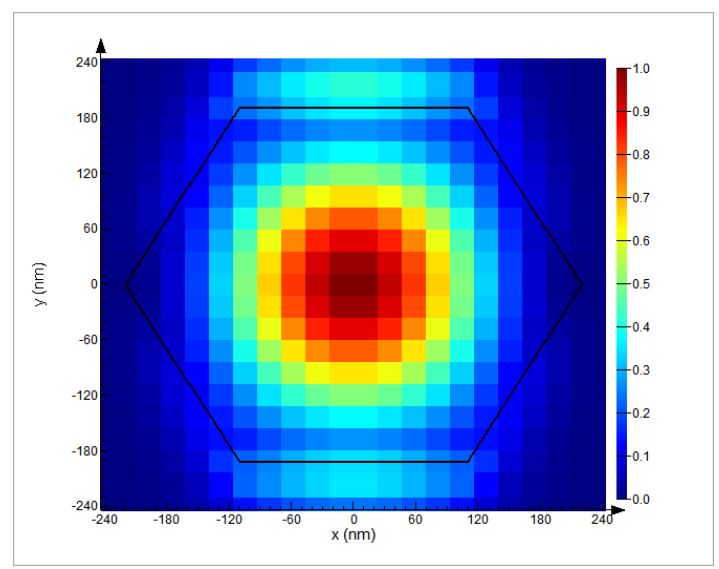

Figure 7: $H E_{11}$ guiding mode in the NW.

To observe the effect of dipole source position on Purcell factor and quality factor, the dipole source is swept from $\mathrm{z}=-4.5 \mathrm{um}$ to $\mathrm{z}=4.5 \mathrm{um}$. The two diagrams obtained from this sweep (Figure 8) change almost symmetrically and three peaks can be seen in it. As shown in Figure 8, when the source is more than 2.5 um away from the center of the NW, the quality factor decreases. However, the Purcell factor did not decrease sharply and did not fluctuate more except at the origin of the NW. The maximum value of Purcell factor is obtained when the dipole source is located in the center of the NW $\left(F_{p} \approx 4.72\right)$. Since the excitation efficiency of NW modes depends on the intensity of the field of a mode at the location of the dipole source, by placing the source in the center of the NW axis, the electric field also intensifies. 


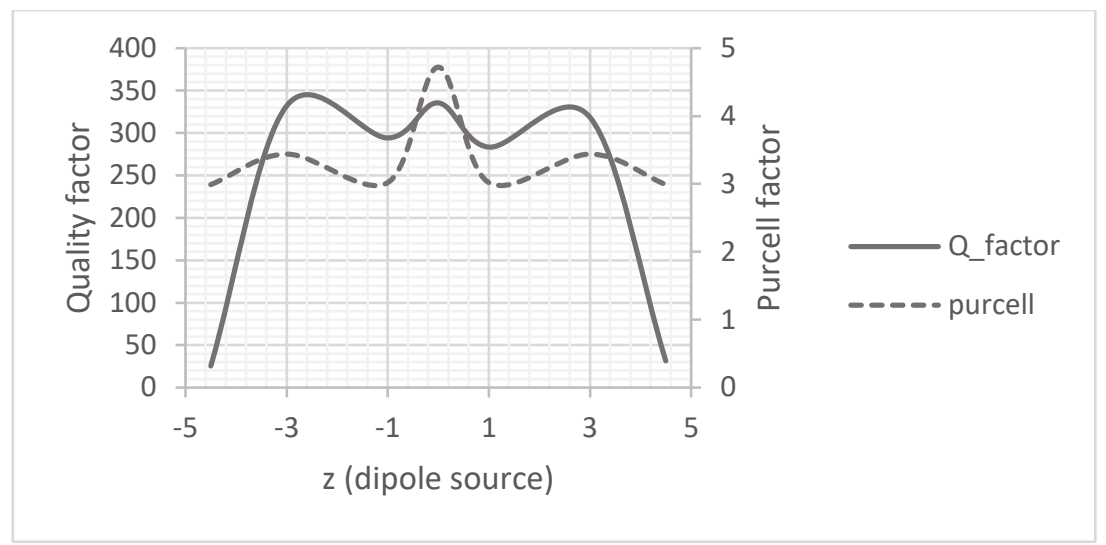

Figure 8: Purcell factor and Quality factor versus variation of the dipole source location along the NW axis.

Although in FDTD simulations the QD is modeled with a dipole source, a QD of PbS material is placed (so that the dipole source is in the center of the QD) and a finer mesh is selected for its structure. Afterwards the effect of the QD material is included in the simulations. The simulation results show that for circle QDs with a radius of $\sim 8 \mathrm{~nm}$, the highest value for Purcell factor is obtained (Figure 9); Although PbS QDs built in practice, are spheres. According to the simulation, by increasing the radius of the sphere QD, the Purcell factor decreases with a gentle slope.

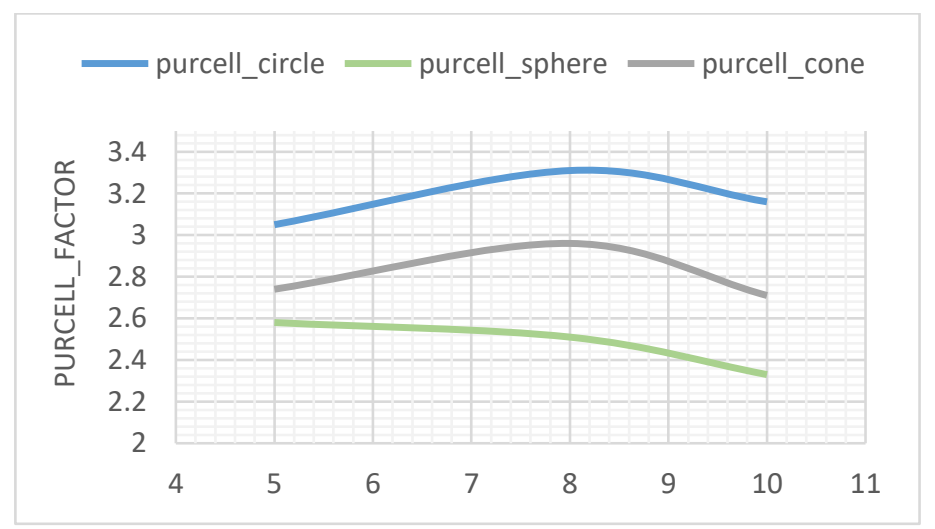

Figure 9: Purcell factor versus radius variations of circle (blue), sphere (green) and cone (gray) type QDs. 
The characteristics of the final structure are summarized in

Table Error! Reference source not found.. The shape of the final structure is also shown in Figure 10.

Table 1. Characteristics of the final simulated structure.

\begin{tabular}{|c|c|}
\hline Characteristics & Value \\
\hline NW radius & $220 \mathrm{~nm}$ \\
\hline NW height & $10 \mathrm{um}$ \\
\hline QD radius & $8 \mathrm{~nm}$ \\
\hline QD shape & circle \\
\hline $\mathrm{Z}(\mathrm{QD})$ & 0 \\
\hline
\end{tabular}
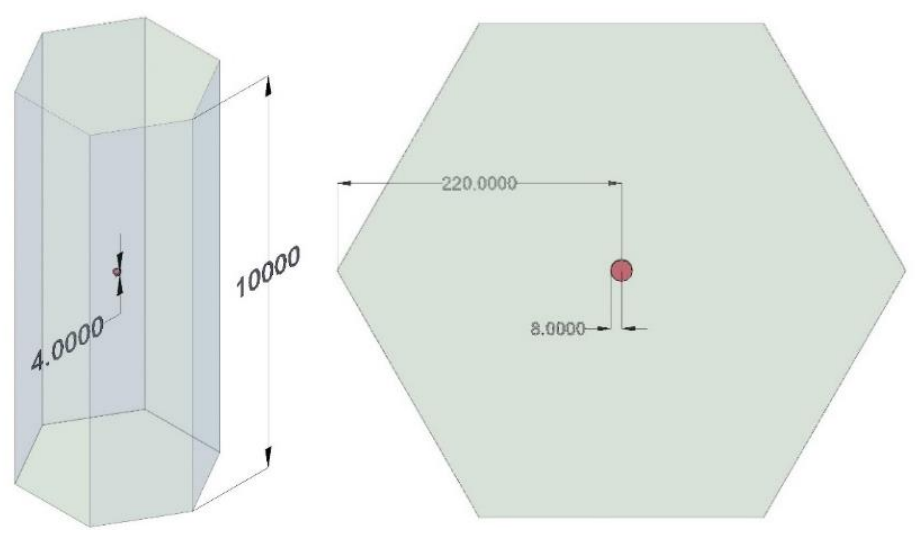

Figure 10: Final simulated QD within NW structure (dimensions are in nanometers).

The Purcell spectrum of the final structure in Figure 11(a) shows that the highest amount of Purcell factor occurs at $1025.4 \mathrm{~nm}$, with a quality factor of 295.25 at this wavelength. The wavelength peak of above lum makes the single photon source useful for quantum communications. The intensity of the electric field at this wavelength is $\sim 15 \mathrm{a}$.u., which is propagated in $H E_{11}$ mode as presented in Figure 11(c). 


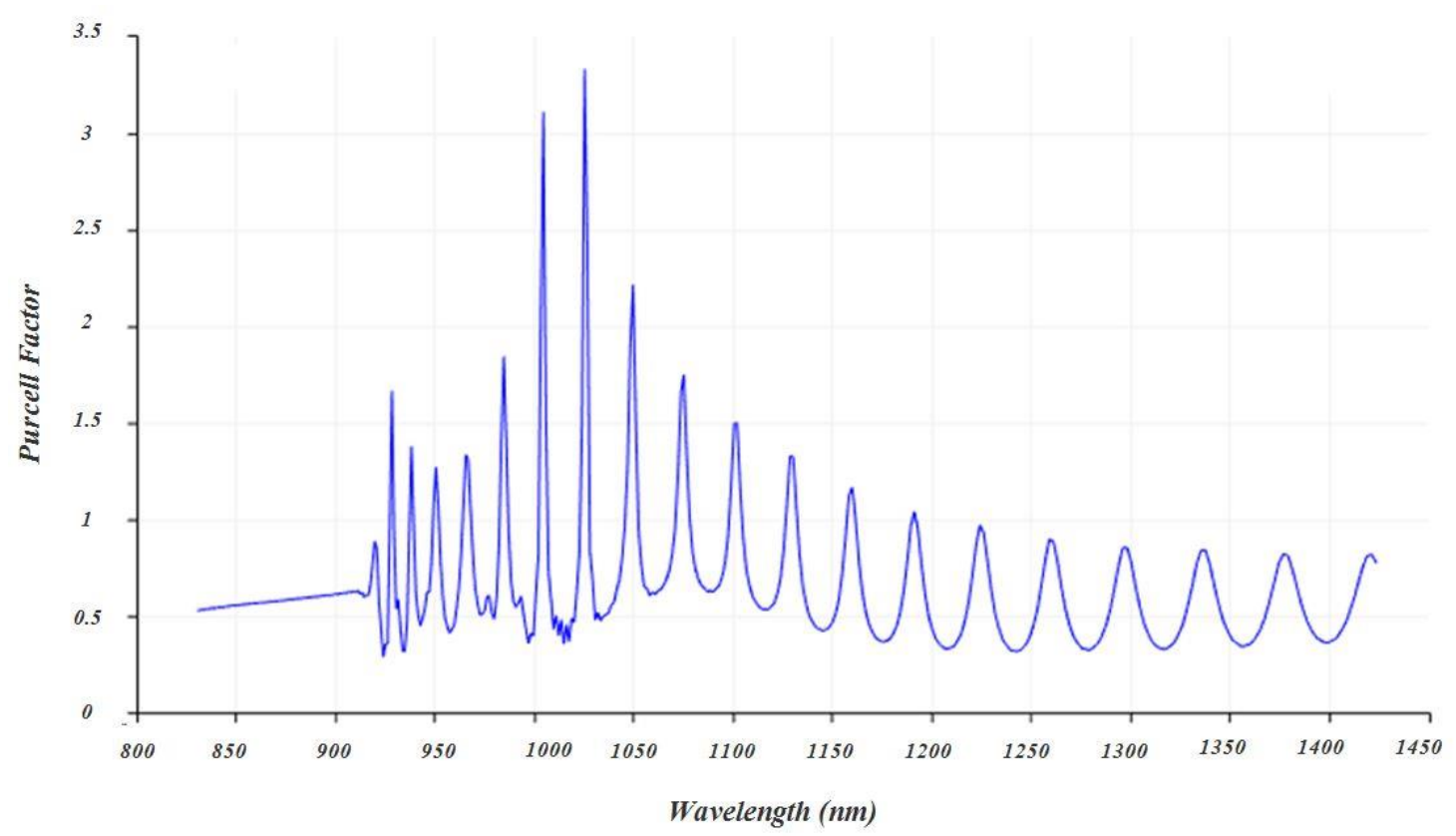

(a)

\section{Photoluminescence Spectrum}

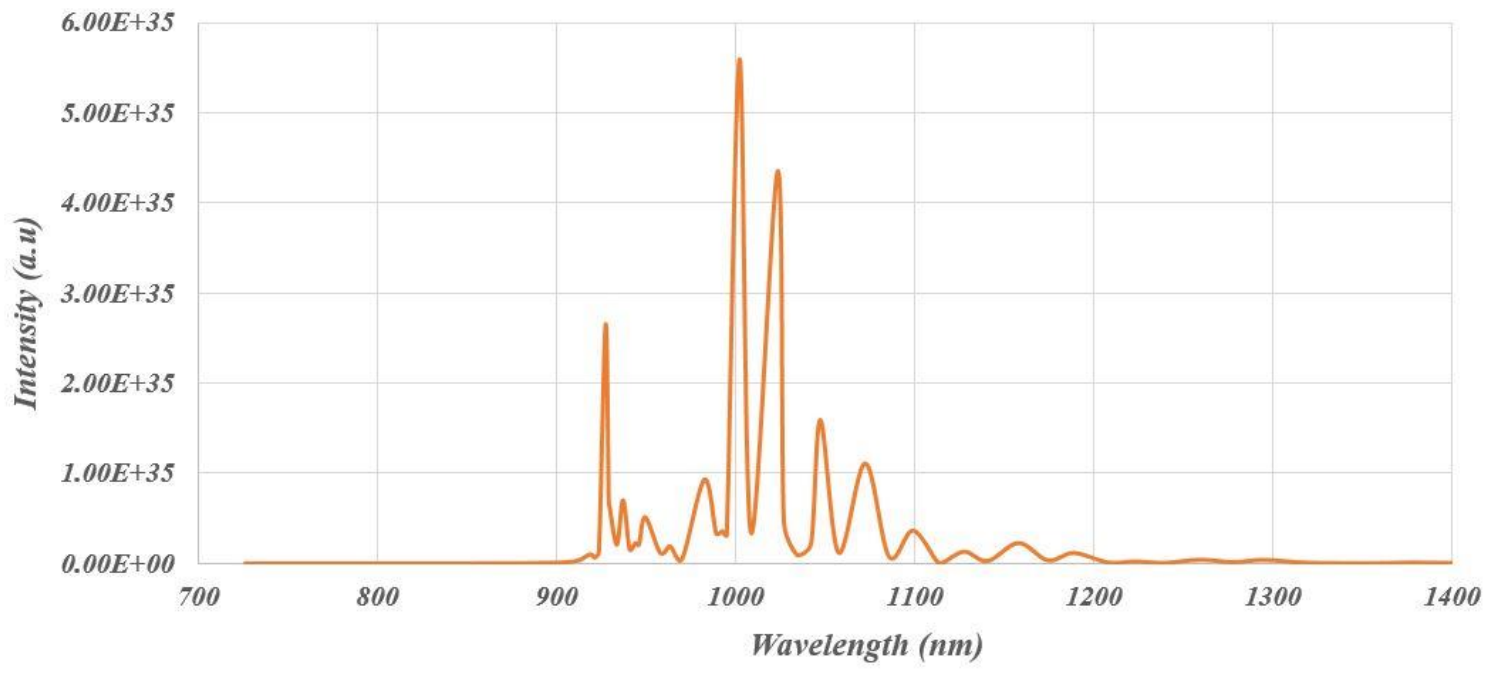

(b) 


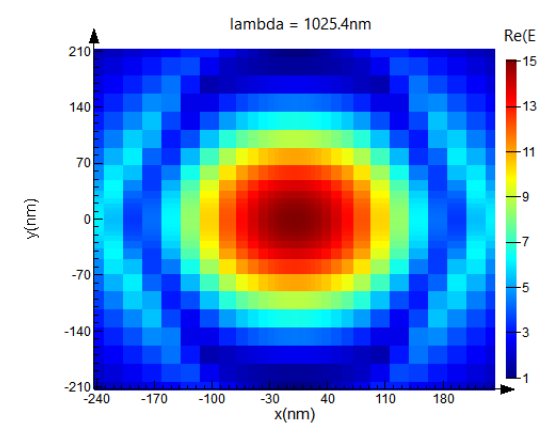

(c)

Figure 11: a) Purcell spectrum, b) Spectra of NW waveguide, c) Electric field of final structure simulated in FDTD.

\section{Conclusion}

In this paper the effect of height and radius of the hexagonal InP nanowire as well as the location and orientation of dipole source in the Purcell factor and Quality factor are investigated. The maximum Purcell factor and Quality factor have obtained for a nanowire with height and radius of 10um and $220 \mathrm{~nm}$ respectively. In addition, it is observed that for a dipole source which located in the center of the nanowire axis with orientation perpendicular to the axis, maximum electric field intensity is obtained. Also, the results have shown we have maximum Purcell factor for $\sim 8 \mathrm{~nm}$ circular $\mathrm{PbS}$ QDs, although they are spherical in practice.

\section{Conflicts of interest}

There are no conflicts of interest to declare. 


\section{References}

[1] T. Nozaka and K. Mukai, "Controlled waveguide coupling for photon emission from colloidal PbS quantum dot using tunable microcavity made of optical polymer and silicon," Physica E: Lowdimensional Systems and Nanostructures, vol. 78, pp. 14-18, 2016.

[2] M. Anderson et al., "Coherence in single photon emission from droplet epitaxy and StranskiKrastanov quantum dots in the telecom C-band," Applied Physics Letters, vol. 118, no. 1, p. 014003, 2021.

[3] A. Baskaran and P. Smereka, "Mechanisms of stranski-krastanov growth," Journal of Applied Physics, vol. 111, no. 4, p. 044321, 2012.

[4] Y. Yu et al., "Self-assembled semiconductor quantum dots decorating the facets of GaAs nanowire for single-photon emission," National Science Review, vol. 4, no. 2, pp. 196-209, 2017.

[5] A. Artioli, S. Kotal, N. Gregersen, P. Verlot, J.-M. Gérard, and J. Claudon, "Design of Quantum Dot-Nanowire Single-Photon Sources that are Immune to Thermomechanical Decoherence," Physical review letters, vol. 123, no. 24, p. 247403, 2019.

[6] R. M. Stevenson, R. J. Young, P. Atkinson, K. Cooper, D. A. Ritchie, and A. J. Shields, "A semiconductor source of triggered entangled photon pairs," Nature, vol. 439, no. 7073, pp. 179$182,2006$.

[7] K. Mukai and K. Nakashima, "Suppression of the polarization dependence of the vertical photoluminescence from InAs/GaAs quantum dots by InGaAs strain-reducing layer," Japanese Journal of Applied Physics, vol. 47, no. 6S, p. 5057, 2008.

[8] K. Mukai, K. Watanabe, and K. Nakashima, "Computational Study on the In-Plane Symmetry of Electron Wavefunctions in Self-Assembled InAs/GaAs Quantum Dots," Journal of nanoscience and nanotechnology, vol. 9, no. 1, pp. 108-114, 2009.

[9] M.-Q. Wang, Y.-Z. Huang, Q. Chen, and Z.-P. Cai, "Analysis of mode quality factors and mode reflectivities for nanowire cavity by FDTD technique," IEEE journal of quantum electronics, vol. 42, no. 2, pp. 146-151, 2006.

[10] B. Lounis and M. Orrit, "Single-photon sources," Reports on Progress in Physics, vol. 68, no. 5, p. $1129,2005$.

[11] G. Cirlin et al., "Hybrid GaAs/AlGaAs Nanowire-Quantum dot System for Single Photon Sources," Semiconductors, vol. 52, no. 4, pp. 462-464, 2018.

[12] M. J. Holmes, K. Choi, S. Kako, M. Arita, and Y. Arakawa, "Room-temperature triggered single photon emission from a III-nitride site-controlled nanowire quantum dot," Nano letters, vol. 14, no. 2, pp. 982-986, 2014.

[13] L. Leandro et al., "Nanowire quantum dots tuned to atomic resonances," Nano letters, vol. 18, no. 11, pp. 7217-7221, 2018.

[14] Y. Yu et al., "Self-Assembled Quantum Dot Structures in a Hexagonal Nanowire for Quantum Photonics," Advanced Materials, vol. 26, no. 17, pp. 2710-2717, 2014.

[15] S. Dorenbos et al., "Position controlled nanowires for infrared single photon emission," Applied Physics Letters, vol. 97, no. 17, p. 171106, 2010.

[16] M. E. Reimer et al., "Bright single-photon sources in bottom-up tailored nanowires," Nature communications, vol. 3, no. 1, pp. 1-6, 2012.

[17] I. Fushman, D. Englund, and J. Vučković, "Coupling of PbS quantum dots to photonic crystal cavities at room temperature," Applied Physics Letters, vol. 87, no. 24, p. 241102, 2005.

[18] G. M. Akselrod, M. C. Weidman, Y. Li, C. Argyropoulos, W. A. Tisdale, and M. H. Mikkelsen, "Efficient nanosecond photoluminescence from infrared $\mathrm{PbS}$ quantum dots coupled to plasmonic nanoantennas," Acs Photonics, vol. 3, no. 10, pp. 1741-1746, 2016.

[19] H. Arab, S. MohammadNejad, A. KhodadadKashi, and S. Ahadzadeh, "Recent advances in nanowire quantum dot (NWQD) single-photon emitters," Quantum Information Processing, vol. 19, no. 2, pp. 1-84, 2020. 
[20] L. Elsinger et al., "Integration of colloidal $\mathrm{PbS} / \mathrm{CdS}$ quantum dots with plasmonic antennas and superconducting detectors on a silicon nitride photonic platform," Nano letters, vol. 19, no. 8, pp. 5452-5458, 2019.

[21] Z. Huang et al., "PbS/CdS core-shell quantum dots suppress charge transfer and enhance triplet transfer," Angewandte Chemie International Edition, vol. 56, no. 52, pp. 16583-16587, 2017.

[22] A.-L. Henneghien, B. Gayral, Y. Désières, and J.-M. Gérard, "Simulation of waveguiding and emitting properties of semiconductor nanowires with hexagonal or circular sections," JOSA $B$, vol. 26, no. 12, pp. 2396-2403, 2009.

[23] C. Downs and T. E. Vandervelde, "Progress in infrared photodetectors since 2000," Sensors, vol. 13, no. 4, pp. 5054-5098, 2013.

[24] X. Fu, Y. Pan, X. Wang, and J. R. Lombardi, "Quantum confinement effects on charge-transfer between $\mathrm{PbS}$ quantum dots and 4-mercaptopyridine," The Journal of chemical physics, vol. 134, no. 2, p. 024707, 2011.

[25] T. Nobis and M. Grundmann, "Low-order optical whispering-gallery modes in hexagonal nanocavities," Physical Review A, vol. 72, no. 6, p. 063806, 2005.

[26] Y. Ma, X. Guo, X. Wu, L. Dai, and L. Tong, "Semiconductor nanowire lasers," Advances in optics and photonics, vol. 5, no. 3, pp. 216-273, 2013.

[27] M. Law, D. J. Sirbuly, J. C. Johnson, J. Goldberger, R. J. Saykally, and P. Yang, "Nanoribbon waveguides for subwavelength photonics integration," Science, vol. 305, no. 5688, pp. 1269-1273, 2004.

[28] L. Tong et al., "Subwavelength-diameter silica wires for low-loss optical wave guiding," Nature, vol. 426, no. 6968, pp. 816-819, 2003. 


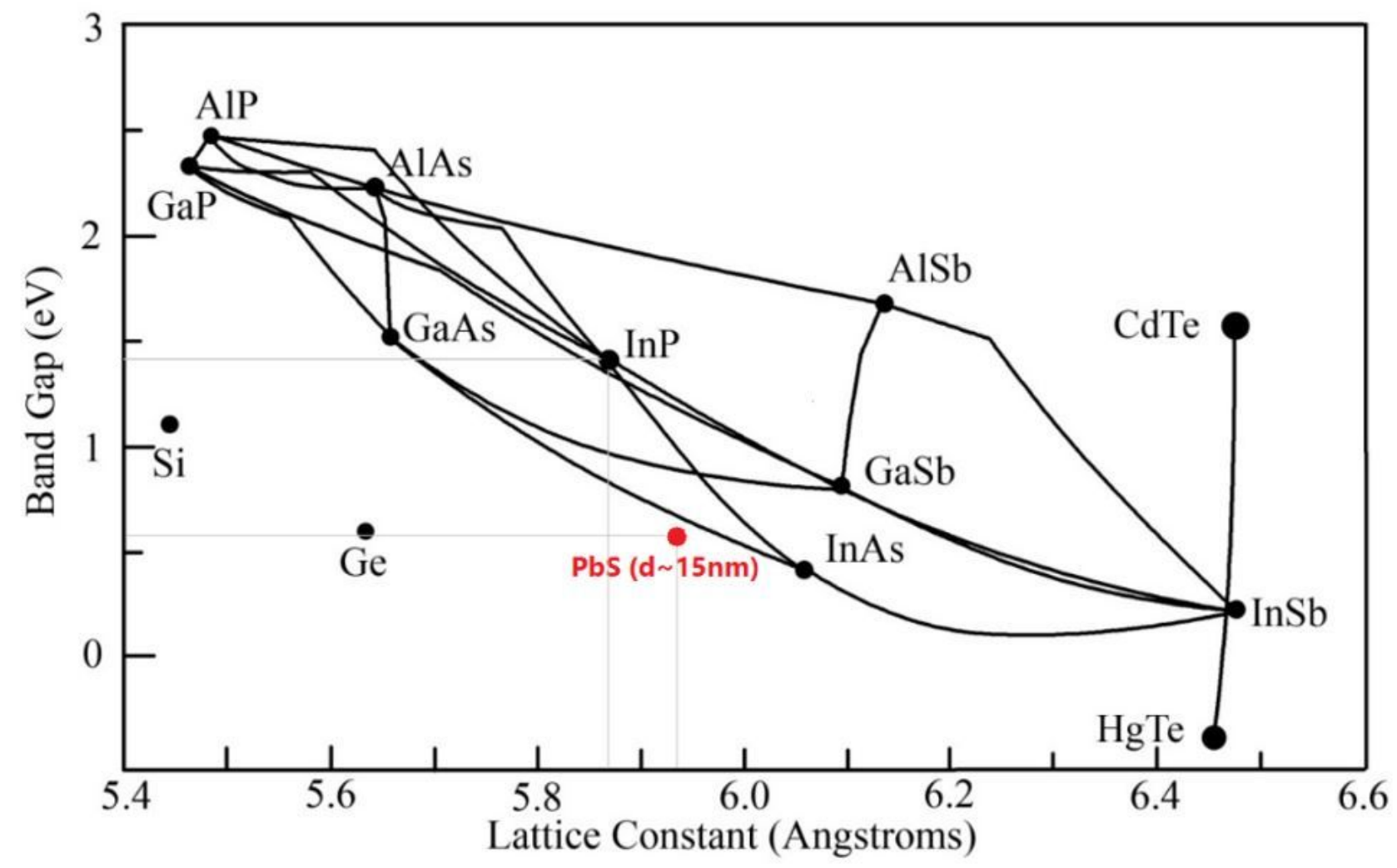

Figure 1

band gap diagram versus lattice constant for some semiconductor materials [23]. Properties for PbS QDs with diameter of $15 \mathrm{~nm}$ have been added (red circle) [24].

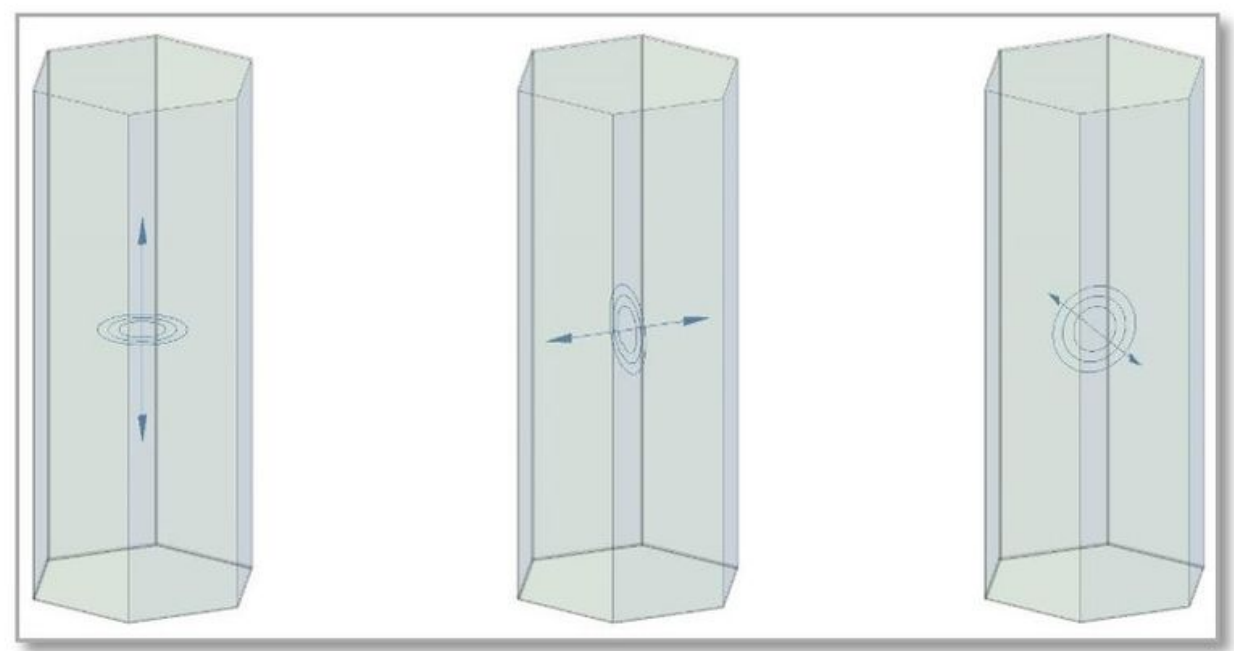

a)

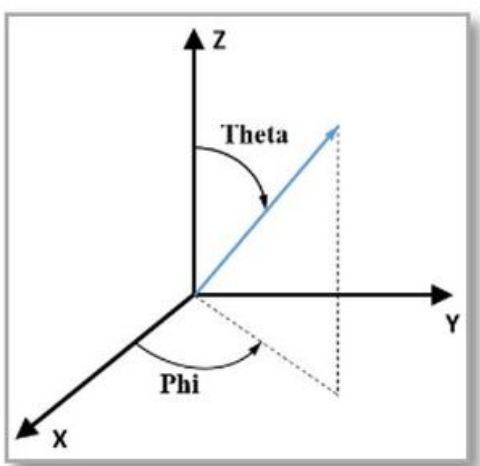

b)

Figure 2 
a) Illustrations of various types of dipole source orientation within the NW structure. b) Theta and phi angles.

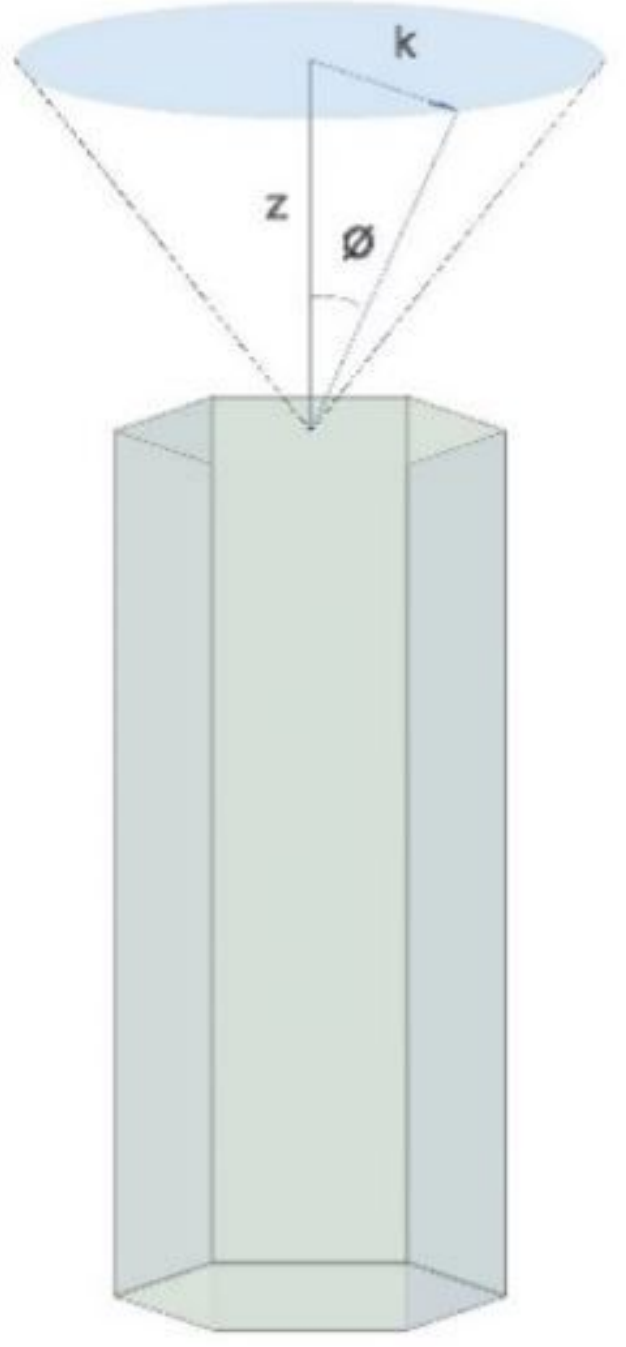

Figure 3

Light transverse k-vector and $\emptyset$ angle. 


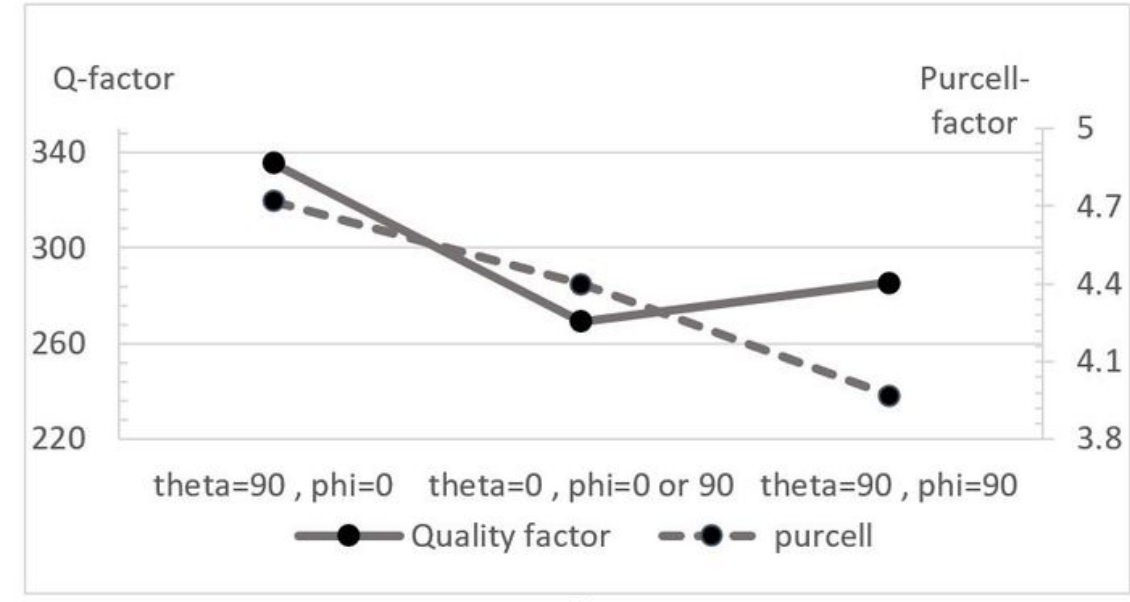

a)

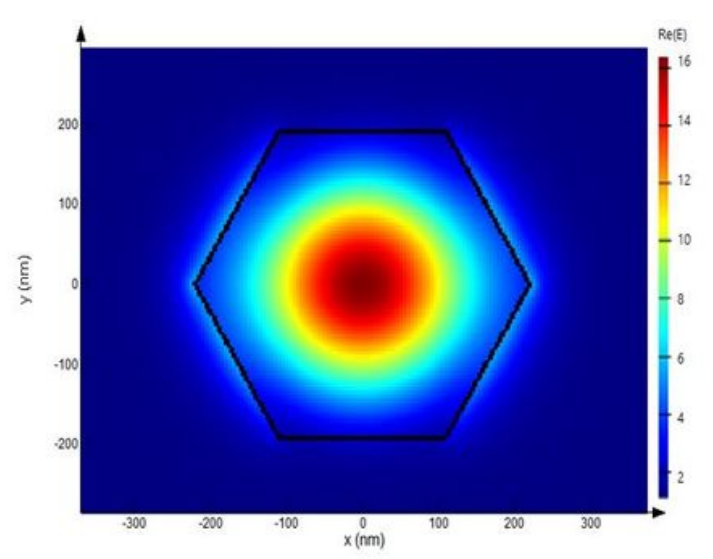

b)

\section{Figure 4}

a) Quality factor and Purcell factor versus variation of polarization angle of dipole source. b) The intensity of electric field per theta $=90$ and phi $=0$.

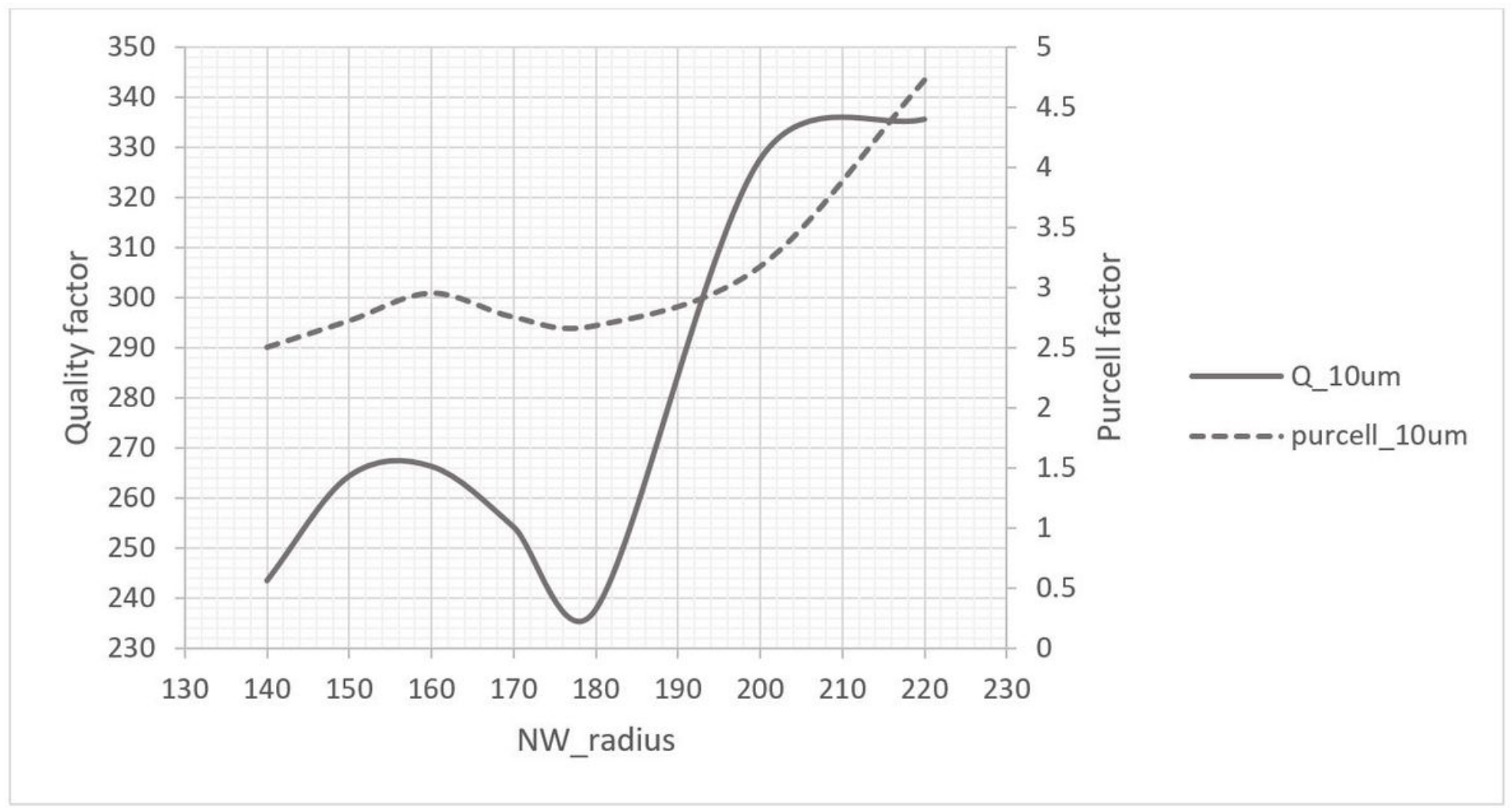

\section{Figure 5}

Purcell factor and Quality factor versus variation of NW radius (the NW height is considered to be 10um). 


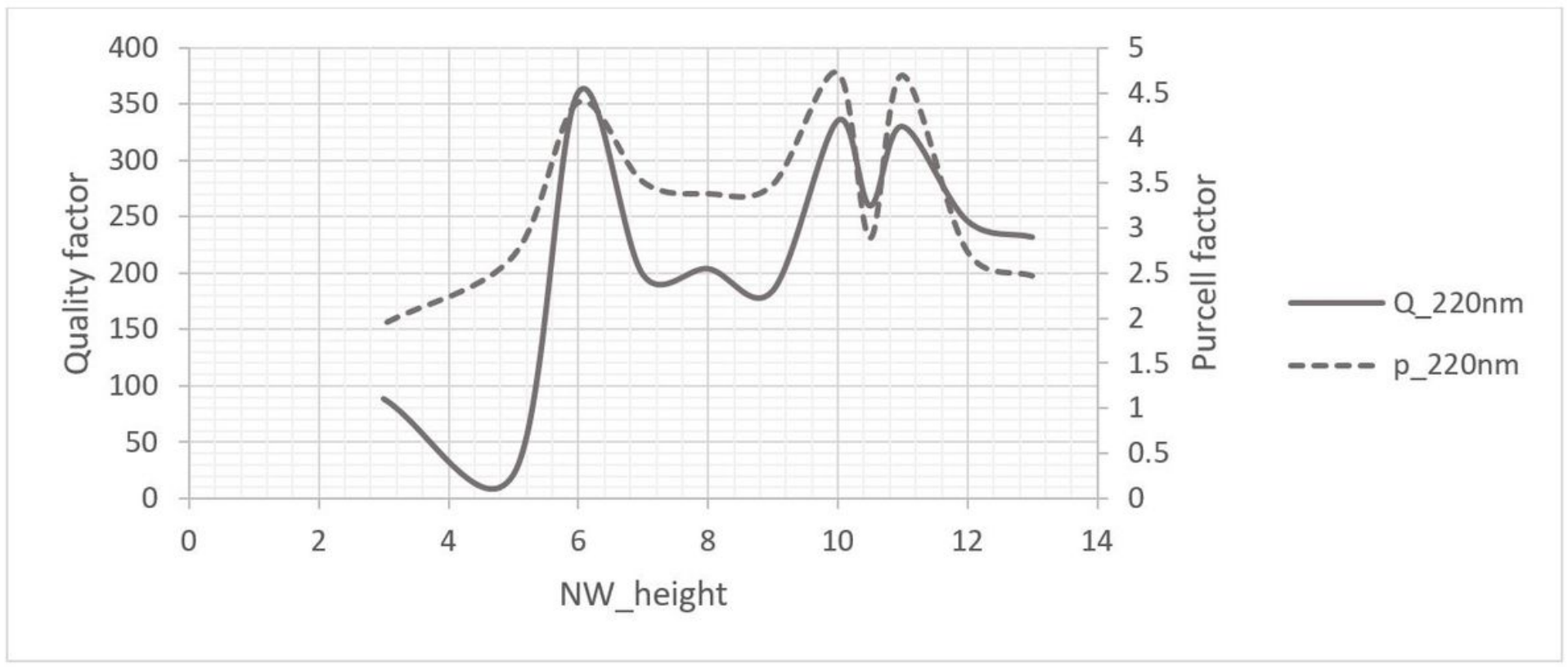

\section{Figure 6}

Purcell factor and Quality factor versus variation of the height of the NW (the NW radius is considered to be $220 \mathrm{~nm})$. 


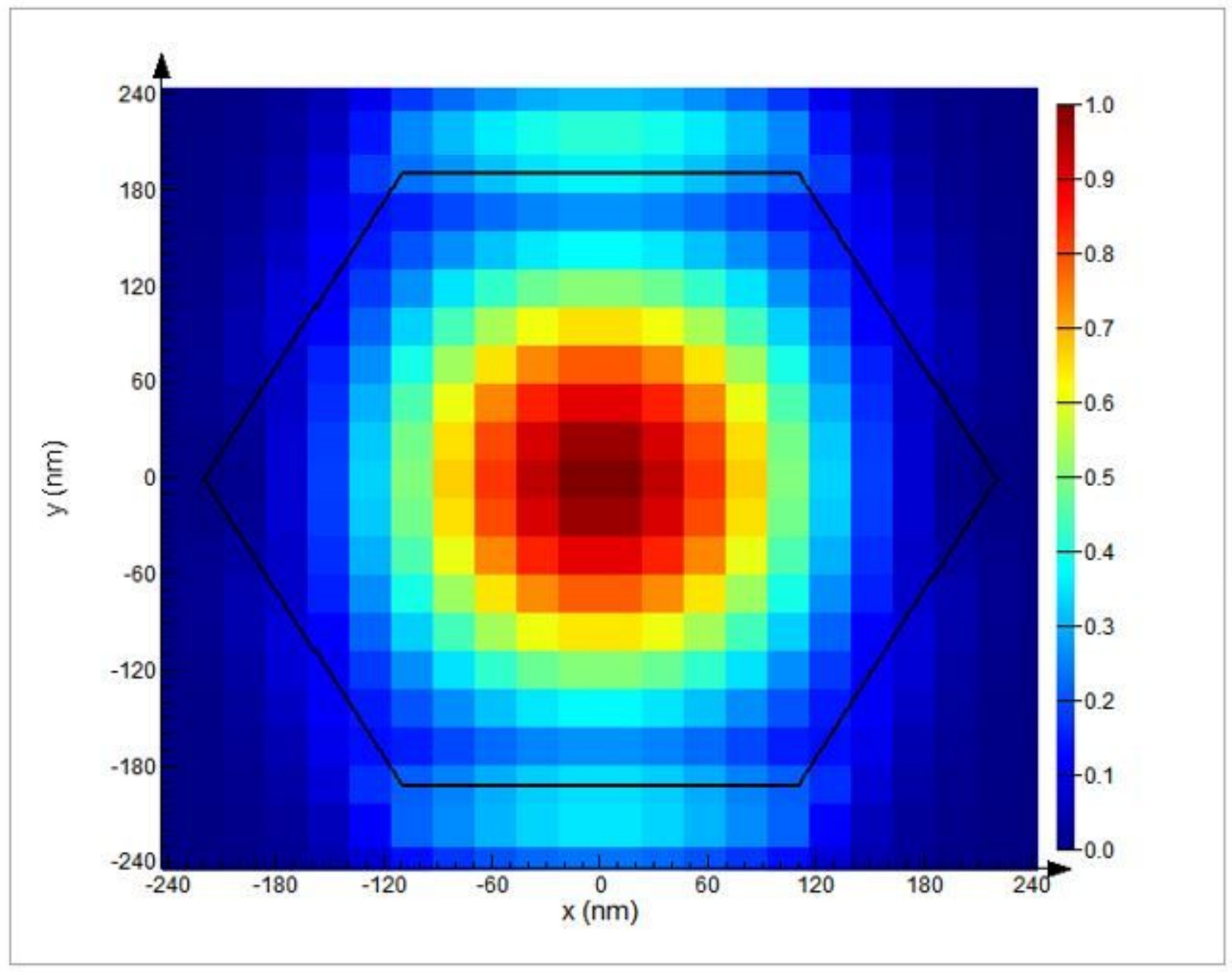

Figure 7

HE11 guiding mode in the NW. 


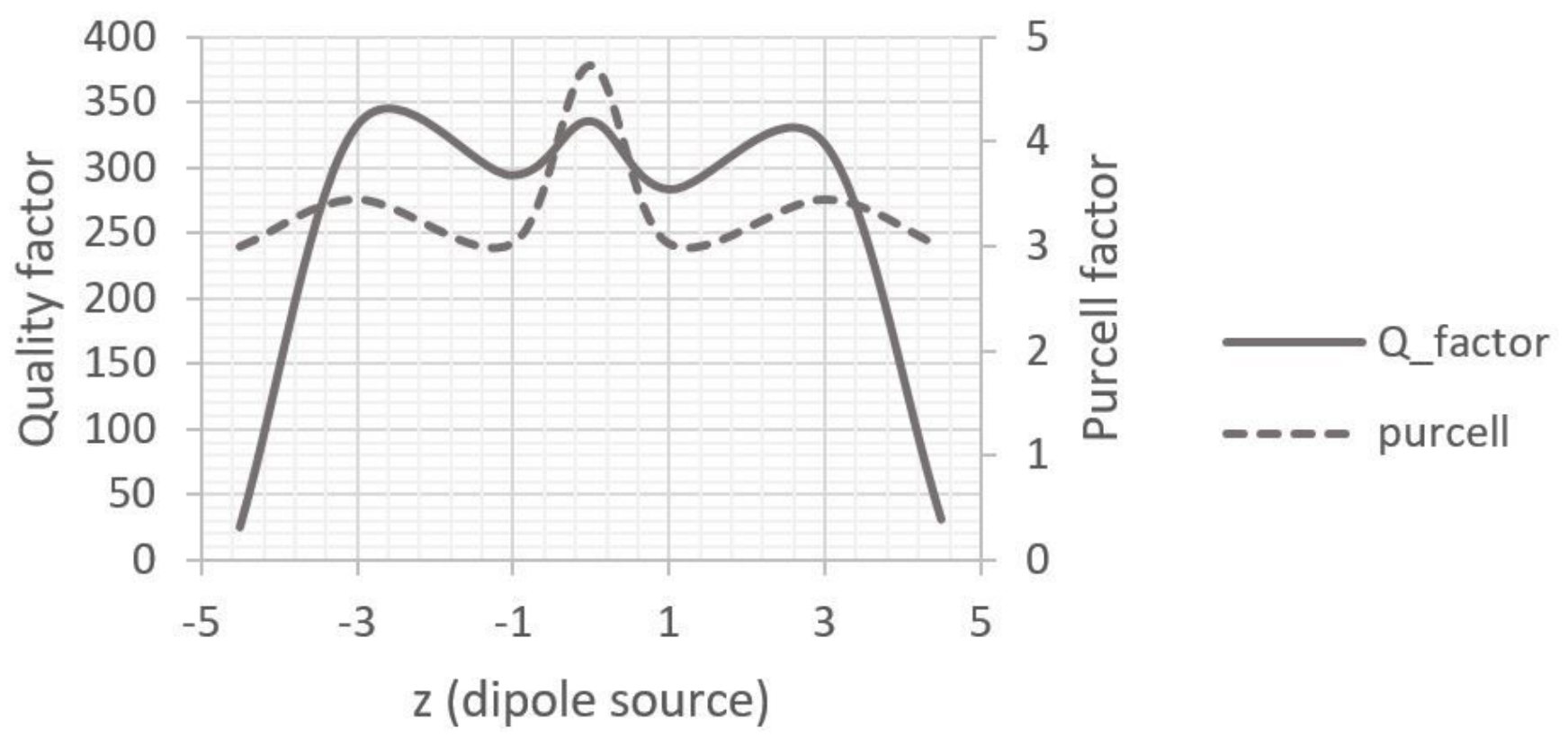

Figure 8

Purcell factor and Quality factor versus variation of the dipole source location along the NW axis.

— purcell_circle purcell_sphere w purcell_cone

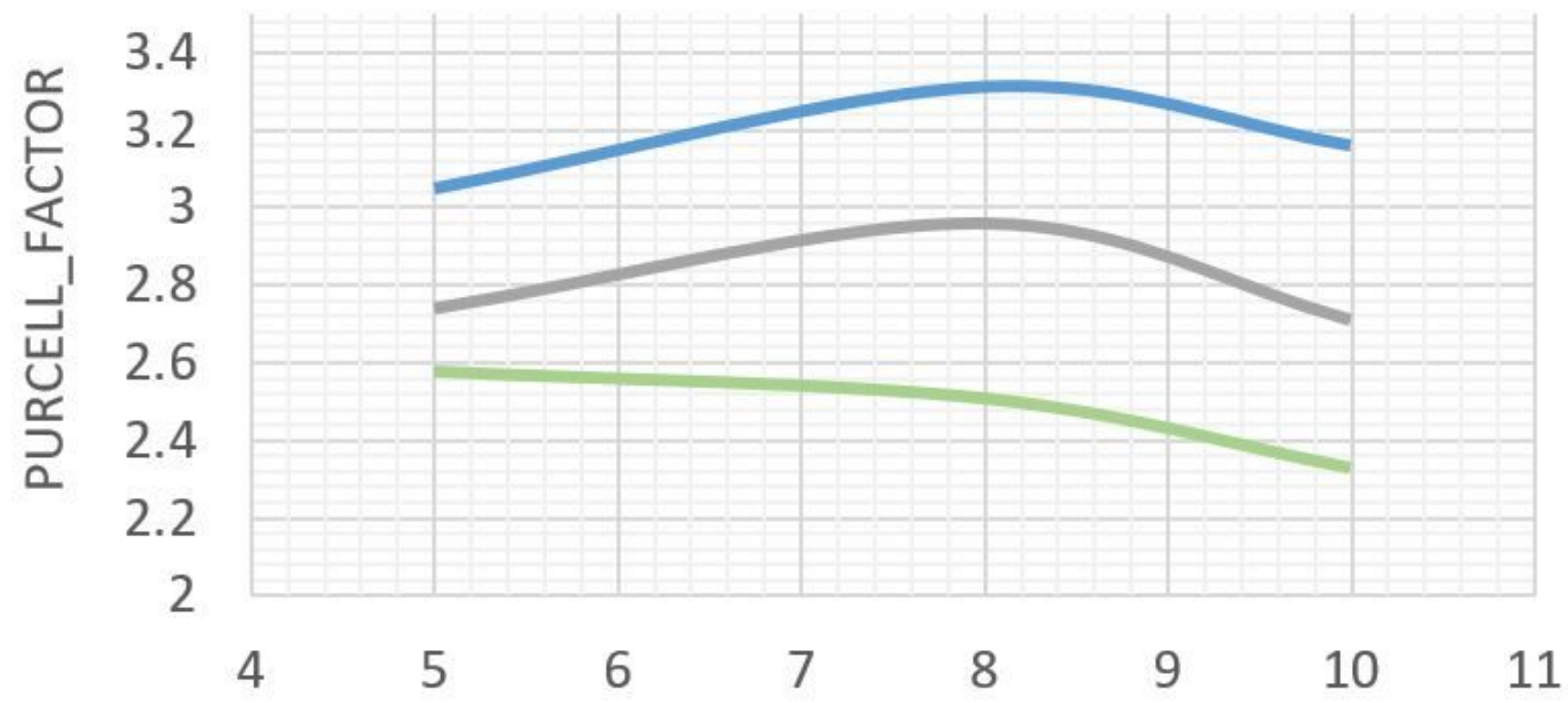

Figure 9 
Purcell factor versus radius variations of circle (blue), sphere (green) and cone (gray) type QDs.
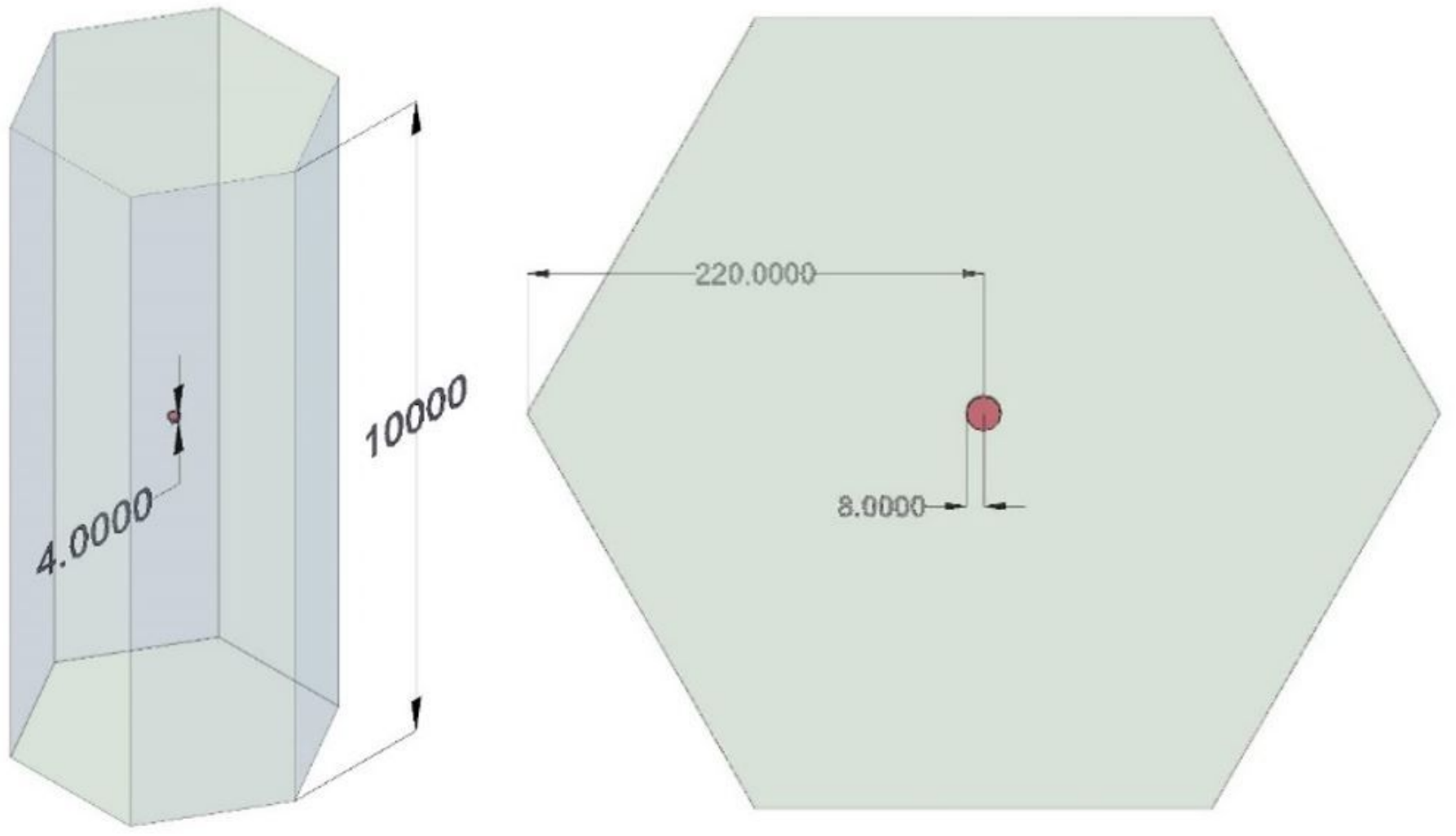

Figure 10

Final simulated QD within NW structure (dimensions are in nanometers). 


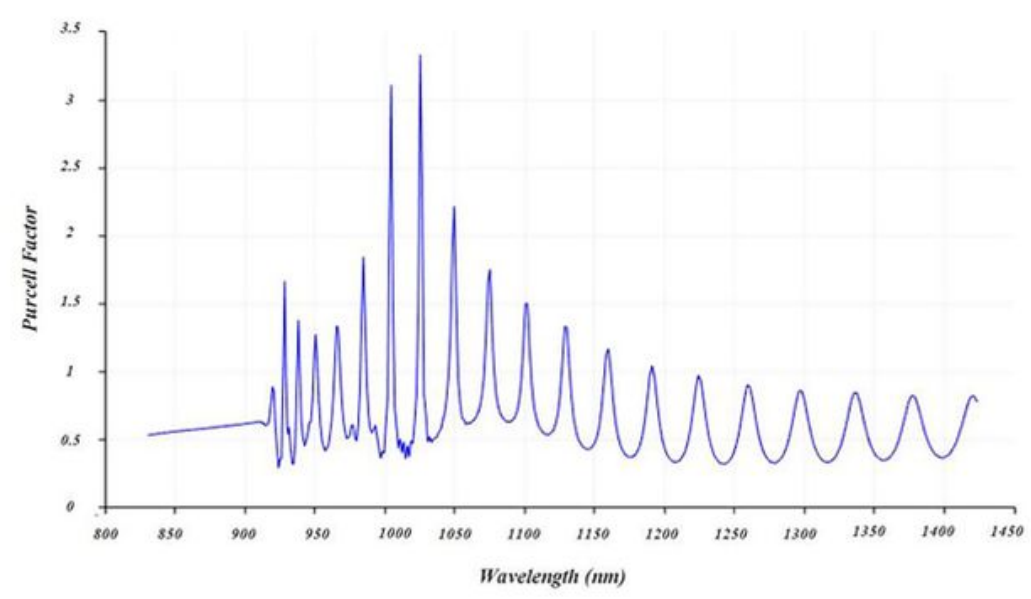

(a)

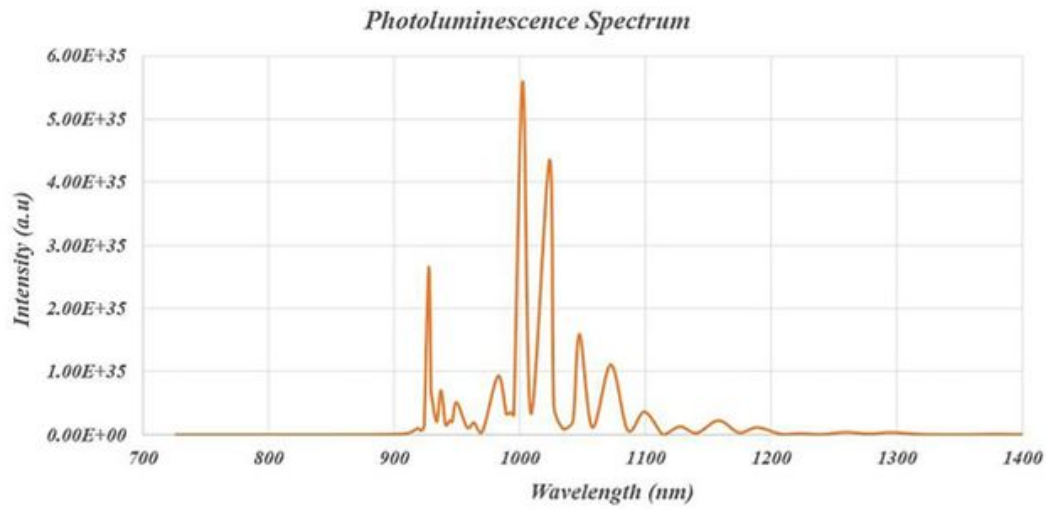

(b)

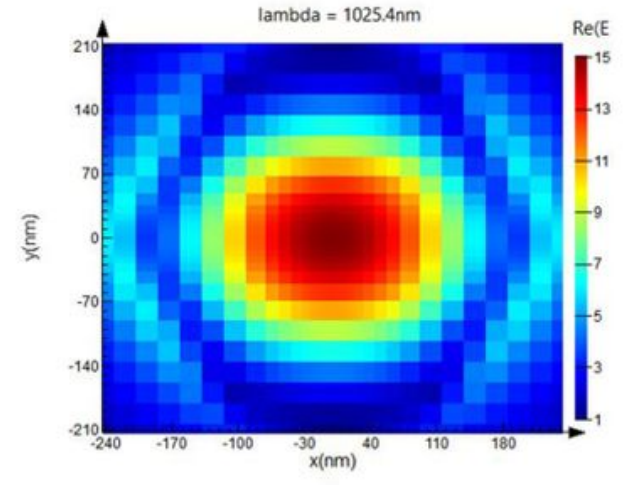

(c)

\section{Figure 11}

a) Purcell spectrum, b) Spectra of NW waveguide, c) Electric field of final structure simulated in FDTD. 